\title{
Uso endoscópico de coagulación con argón plasma en obstrucción benigna de la vía aérea central. Análisis de una serie de casos
}

\author{
Pablo Álvarez-Maldonado, * Benito Vargas-Abrego,* Francisco Navarro-Reynoso,* \\ Carlos Núñez-Pérez Redondo,*,ه Raúl Cicero-Sabido*,*
}

*Hospital General de México “Dr. Eduardo Liceaga”, Ciudad de México; ${ }^{\ddagger}$ Facultad de Medicina, UNAM. Trabajo recibido: 12-X-2015; aceptado: 02-XII-2015

\begin{abstract}
RESUMEN. Introducción: Tratar la obstrucción benigna de la vía aérea (OBVA) continúa siendo un reto. Describimos resultados del uso de coagulación con argón plasma (CAP) en OBVA. Métodos: Análisis retrospectivo de una serie de casos. Resultados: Se incluyeron 20 pacientes con OBVA tratados en dos años, con edad promedio $38.9 \pm 12.8$ años, 12 fueron mujeres. La OBVA fue secundaria a: intubación (n $=10)$, infección $(n=4)$, traqueoplastia $(n=2)$, lobectomía $(n=1)$, reacción inflamatoria por cuerpo extraño $(n=1)$, granulomatosis de Wegener $(n=1)$ y trauma $(n=1)$. La localización de la obstrucción fue en subglotis $(70 \%)$, en tráquea en tercio superior $(10 \%)$ y tercio medio $(10 \%)$, en bronquio principal izquierdo (5\%) y bronquio principal derecho (5\%). Se utilizó CAP en un total de 38 sesiones (rango 1-7 sesiones/paciente); además, se realizó dilatación laríngea y/o traqueal en 11 casos. En 11 casos hubo mejoría significativa del grado de oclusión, en 8 la mejoría fue mínima y en 1 no hubo cambios. El seguimiento promedio fue de $15.7 \pm 6.4$ meses (rango 7-30). No hubo complicaciones relacionadas a los procedimientos. Conclusiones: La OBVA puede ser tratada de manera efectiva y segura con CAP en casos seleccionados. El manejo combinado con dilatadores puede ser de ayuda para disminuir el grado de obstrucción.
\end{abstract}

Palabras clave: Coagulación con argón plasma, obstrucción benigna, vía aérea, tráquea, bronquios.

ABSTRACT. Introduction: Benign central airway obstruction (BCAO) management remains a challenge. We describe the results with the use of argon plasma coagulation (APC) in BCAO. Methods: Retrospective case series analysis. Results: Twenty patients with BCAO were treated in a two-year period. With a mean age of $38.9 \pm 12.8$ years; twelve were women. BCAO was secondary to intubation $(n=10)$, infection $(n=4)$, tracheoplasty $(n=2)$; lobectomy $(n=1)$; inflammatory response to foreign body $(n=1)$; Wegener's granulomatosis $(n=1)$; and trauma $(n=1)$. Location of the obstruction was at the subglottic space in $70 \%$; upper third of the trachea in $10 \%$; half third of the trachea in $10 \%$; left main bronchi in 5\%; and right main bronchi in 5\%. CAP was used for a total of 38 sessions (range 1-7 sessions/patient). Also, laryngeal and/ or tracheal dilatation was performed in eleven cases. Significant improvement was observed in eleven cases, while eight cases experimented minimal improvement and one case did not improve at all. Mean patient follow-up was $15.7 \pm 6.4$ months (range 7-30). There were no complications related to the procedures. Conclusions: In selected cases, BCAO could be effectively and safely treated by means of CAP. Combined treatment with dilators could be of helpful in recovering the occluded airway.

Key words: Argon plasma coagulation, benign obstruction, airways, trachea, bronchi.

\section{INTRODUCCIÓN}

La obstrucción benigna de la vía aérea central (OBVA) afecta la laringe, tráquea y/o bronquios principales. La causa más frecuente es la intubación traqueal, sea prolongada o no., ${ }^{1,2}$ Otras causas menos frecuentes incluyen infecciones (principalmente las causadas por Mycobacterium tuberculosis y Klebsiella escleromatis), enfermedades autoinmunes, trauma directo y cirugía previa laringotraqueal o bronquial. ${ }^{3,4}$ Por su complejidad, la OBVA se considera un problema grave de salud. La respuesta al tratamiento es muy variable y la recidiva puede presentarse independiente de la etiología. . $^{5-7}$

El manejo de la OBVA sigue siendo poco comprendido e impone un reto a cirujanos y neumólogos intervencionistas. Aunque la reconstrucción quirúrgica es considerada por muchos el abordaje terapéutico ideal, los pacientes deben someterse a una evaluación cuidadosa para su selección y ser intervenidos por un equipo quirúrgico experimentado para obtener los 
mejores resultados. ${ }^{8-10}$ El tratamiento endoscópico es una alternativa viable también en casos seleccionados. ${ }^{11}$ Las opciones de tratamiento endoscópico de la OBVA incluyen la dilatación mecánica con dilatadores secuenciales o con balón, y la resección mecánica o por ablación con electrocoagulación, láser o crioterapia, entre otros..$^{12,13}$ Ambos, dilatación y resección pueden llevarse a cabo individualmente o en conjunto con el uso posterior de adyuvantes como corticosteroides, mitomicina-C o stents para mantener permeable el lumen de la vía aérea que se ha recuperado. ${ }^{14}$

El tratamiento endoscópico de la OBVA con coagulación con argón plasma (CAP) por medio de broncoscopia flexible, es una técnica relativamente reciente. Describimos la experiencia de dos años en el tratamiento de OBVA con CAP en una serie de casos de un hospital de tercer nivel en México.

\section{MATERIAL Y MÉTODOS}

Diseño: Estudio de serie de casos, retrospectivo, descriptivo.

Pacientes y lugar: Se incluyeron pacientes adultos que ingresaron al Servicio de Neumología y Cirugía de Tórax del Hospital General de México «Dr. Eduardo Liceaga» (Hospital Universitario de Concentración de Tercer Nivel) entre el $1^{\circ}$ de enero de 2011 y el $1^{\circ}$ de enero de 2013, que fueron diagnosticados con OBVA por medio de broncoscopia flexible. Los datos fueron recabados de manera directa del expediente clínico hospitalario y del expediente broncoscópico de la Unidad de Endoscopia Torácica.

Definiciones: Con información obtenida del expediente broncoscópico, el grado y localización de la obstrucción fue clasificada según Freitag et al. ${ }^{15}$ tal como se describe en la tabla 1. Se consideró que existía mejoría satisfactoria después del tratamiento si había recuperación del lumen de la vía aérea con disminución de por lo menos un grado en la clasificación de Freitag asociada a mejoría de los síntomas.

Análisis estadístico: Se usó estadística descriptiva. Los datos recolectados fueron procesados usando SPSS versión 15 (SPSS Inc., Chicago, IL, USA). Las variables cuantitativas se expresaron en medias aritméticas \pm desviación estándar (DE) o mediana y rango intercuartil cuando corresponde. Las variables cualitativas son expresadas en frecuencias y porcentajes (\%).

Aprobación ética: Por las características del estudio (retrospectivo y descriptivo) la aprobación del Comité de Ética para su desarrollo no fue requerida. Durante la revisión de expedientes, en todos los pacientes se corroboró la presencia de consentimiento informado que autorizó el procedimiento endoscópico motivo del estudio. La confidencialidad de los pacientes fue respetada.

\section{RESULTADOS}

Durante los dos años de estudio fueron diagnosticados 68 casos de OBVA de los cuales, 20 fueron tratados con CAP. La edad promedio de los pacientes fue de $38.9+$ 12.8 años (rango 18 a 68), 12 (60\%) fueron mujeres. La OBVA fue secundaria a intubación en 10 casos (50\%); infección en 4 casos de estenosis traqueal (20\%), 2 secundarias a tuberculosis y 2 a escleroma; traqueoplastia y laringotraqueoplastia en 2 casos (10\%); lobectomía superior izquierda en un caso de obstrucción de bronquio principal izquierdo (5\%); reacción inflamatoria por cuerpo extraño en un caso de aspiración de fragmento de proyectil al bronquio principal derecho (5\%), granulomatosis de Wegener en uno $(5 \%)$ y trauma penetrante de tráquea en un caso (5\%). La localización de la obstrucción fue en subglotis en $70 \%$, tercio superior de tráquea en $10 \%$, tercio medio de tráquea en $10 \%$, bronquio principal izquierdo en $5 \%$ y bronquio principal derecho en $5 \%$. La tabla 2 muestra las características

Tabla 1. Clasificación del grado y localización de la obstrucción según Freitag et al.15

\begin{tabular}{|c|c|c|c|c|c|c|}
\hline & \multicolumn{6}{|c|}{ Grado } \\
\hline & 1 & 2 & 3 & 4 & 5 & -- \\
\hline \multirow[t]{3}{*}{ Oclusión de la luz de la vía aérea (\%) } & $\leq 25 \%$ & $26-50 \%$ & $51-75 \%$ & $76-90 \%$ & $>90 \%$ & -- \\
\hline & \multicolumn{6}{|c|}{ Nivel } \\
\hline & 1 & II & III & IV & V & VI \\
\hline Localización & Subglotis & $\begin{array}{l}\text { Tercio } \\
\text { superior de } \\
\text { tráquea }\end{array}$ & $\begin{array}{c}\text { Tercio medio } \\
\text { de tráquea }\end{array}$ & $\begin{array}{l}\text { Tercio inferior } \\
\text { de tráquea }\end{array}$ & $\begin{array}{l}\text { Bronquio prin- } \\
\text { cipal derecho }\end{array}$ & $\begin{array}{l}\text { Bronquio } \\
\text { principal } \\
\text { izquierdo }\end{array}$ \\
\hline
\end{tabular}


clínicas de los pacientes incluidos y los resultados. Se utilizó CAP en todos los pacientes en un total de 38 sesiones (rango 1-7 sesiones por paciente) usando un broncoscopio flexible (Olympus ${ }^{\circledR}$ BF-1T180, Optical Co., Ltd., Tokyo, Japan) y equipo para CAP (Söring Arco 3000, Medizintechnik Germany), con sondas para argón de $1.8 \mathrm{~mm}$ (Argo-Flex-Prove, Medizintechik, Germany), una potencia programada de $25 \mathrm{~W}$ y flujo de argón de
0.2 a $0.3 \mathrm{~L} / \mathrm{min}$. Además, se realizó dilatación secuencial con dilatadores esofágicos de Jackson o cánulas orotraqueales de Rush de diferentes diámetros en 11 casos de estenosis laríngea o traqueal. En 23 de las 38 sesiones de CAP se usó anestesia general, en el resto anestesia tópica con lidocaína al $2 \%$ y sedación consiente con midazolam (5 mg, i.v.) y (fentanilo $150 \mu \mathrm{g}$, i.v.). Todos los pacientes recibieron dexametasona (8

Tabla 2. Características de los pacientes incluidos y respuesta al tratamiento.

\begin{tabular}{|c|c|c|c|c|c|c|c|c|c|c|c|c|}
\hline $\begin{array}{l}\text { Pac. } \\
\text { Núm. }\end{array}$ & Edad & Sexo & $\begin{array}{c}\text { Causa de la } \\
\text { OBVA }\end{array}$ & $\begin{array}{l}\text { Localiza- } \\
\text { ción }^{\star}\end{array}$ & $\begin{array}{l}\text { Tra- } \\
\text { queos- } \\
\text { tomía } \\
\text { previa }\end{array}$ & $\begin{array}{c}\text { Traqueo- } \\
\text { plastia } \\
\text { previa }\end{array}$ & $\begin{array}{l}\text { Sesiones } \\
\text { de CAP }\end{array}$ & $\begin{array}{c}\text { Sesio- } \\
\text { nes de } \\
\text { dilatación } \\
\text { mecánica }\end{array}$ & $\begin{array}{l}\text { Grado } \\
\text { inicial de } \\
\text { obstruc- } \\
\text { ción* }\end{array}$ & $\begin{array}{l}\text { Grado } \\
\text { final de } \\
\text { obstruc- } \\
\text { ción }^{*}\end{array}$ & $\begin{array}{l}\text { Segui- } \\
\text { miento, } \\
\text { meses }\end{array}$ & Resultado \\
\hline 1 & 37 & $F$ & $\begin{array}{c}\text { Postraqueo- } \\
\text { plastia }\end{array}$ & 1 & No & Sí & 2 & 2 & 4 & 2 & 30 & Satisfactorio \\
\hline 2 & 68 & M & $\begin{array}{l}\text { Posintuba- } \\
\text { ción }\end{array}$ & III & Sí & No & 5 & 4 & 3 & 1 & 27 & Satisfactorio \\
\hline 3 & 28 & $\mathrm{~F}$ & $\begin{array}{l}\text { Posintuba- } \\
\text { ción }\end{array}$ & III & No & No & 3 & 1 & 3 & 1 & 22 & Satisfactorio \\
\hline 4 & 18 & $\mathrm{~F}$ & $\begin{array}{l}\text { Posintuba- } \\
\text { ción }\end{array}$ & 1 & Sí & No & 1 & 2 & 5 & 4 & 22 & $\begin{array}{c}\text { No } \\
\text { satisfactorio }\end{array}$ \\
\hline 5 & 42 & $\mathrm{~F}$ & Infección & II & No & No & 2 & 1 & 3 & 2 & 21 & $\begin{array}{c}\text { No } \\
\text { satisfactorio }\end{array}$ \\
\hline 6 & 46 & $\mathrm{~F}$ & Infección & 1 & Sí & No & 3 & 3 & 2 & 2 & 20 & $\begin{array}{c}\text { No } \\
\text { satisfactorio }\end{array}$ \\
\hline 7 & 25 & $F$ & $\begin{array}{l}\text { Posintuba- } \\
\text { ción }\end{array}$ & 1 & Sí & Sí & 1 & 1 & 4 & 4 & 18 & $\begin{array}{c}\text { No } \\
\text { satisfactorio }\end{array}$ \\
\hline 8 & 53 & M & Trauma & 1 & No & No & 1 & 0 & 5 & 3 & 18 & Satisfactorio \\
\hline 9 & 54 & $\mathrm{~F}$ & $\begin{array}{l}\text { Posintuba- } \\
\text { ción }\end{array}$ & 1 & Sí & No & 1 & 0 & 2 & 1 & 17 & Satisfactorio \\
\hline 10 & 25 & $\mathrm{~F}$ & $\begin{array}{l}\text { Posintuba- } \\
\text { ción }\end{array}$ & 1 & No & No & 2 & 3 & 3 & 1 & 16 & Satisfactorio \\
\hline 11 & 29 & $M$ & $\begin{array}{l}\text { Posintuba- } \\
\text { ción }\end{array}$ & 1 & No & No & 2 & 4 & 2 & 2 & 16 & $\begin{array}{c}\text { No } \\
\text { satisfactorio }\end{array}$ \\
\hline 12 & 46 & $F$ & $\begin{array}{l}\text { Poslobecto- } \\
\text { mía LSI }\end{array}$ & VI & No & No & 1 & 0 & 1 & 0 & 14 & Satisfactorio \\
\hline 13 & 39 & M & $\begin{array}{l}\text { Posintuba- } \\
\text { ción }\end{array}$ & 1 & Sí & No & 1 & 0 & 5 & 2 & 13 & $\begin{array}{c}\text { No } \\
\text { satisfactorio }\end{array}$ \\
\hline 14 & 39 & M & $\begin{array}{l}\text { Postraqueo- } \\
\text { plastia }\end{array}$ & II & No & Sí & 7 & 0 & 4 & 1 & 10.5 & Satisfactorio \\
\hline 15 & 29 & M & $\begin{array}{c}\text { Reacción } \\
\text { a cuerpo } \\
\text { extraño }\end{array}$ & V & No & No & 1 & 0 & 1 & 0 & 10 & Satisfactorio \\
\hline 16 & 31 & $M$ & $\begin{array}{l}\text { Posintuba- } \\
\text { ción }\end{array}$ & 1 & Sí & No & 1 & 1 & 4 & 4 & 9 & $\begin{array}{c}\text { No } \\
\text { satisfactorio }\end{array}$ \\
\hline 17 & 45 & $\mathrm{~F}$ & Infección & 1 & No & No & 2 & 0 & 3 & 1 & 9 & Satisfactorio \\
\hline 18 & 50 & $\mathrm{~F}$ & $\begin{array}{l}\text { Granulo- } \\
\text { matosis de } \\
\text { Wegener }\end{array}$ & 1 & No & No & 1 & 0 & 3 & 3 & 8 & $\begin{array}{c}\text { No } \\
\text { satisfactorio }\end{array}$ \\
\hline 19 & 23 & $M$ & $\begin{array}{l}\text { Posintuba- } \\
\text { ción }\end{array}$ & 1 & No & No & 1 & 0 & 4 & 4 & 7 & $\begin{array}{c}\text { No } \\
\text { satisfactorio }\end{array}$ \\
\hline 20 & 51 & $\mathrm{~F}$ & Infección & 1 & No & No & 1 & 1 & 4 & 2 & 7 & Satisfactorio \\
\hline
\end{tabular}

OBVA = Obstrucción benigna de la vía aérea; CAP = Coagulación con argón plasma; LSI = Lóbulo superior izquierdo. *Según la clasificación de Freitag et al. ${ }^{15}$ 
mg, i.v.) cada 8 horas durante 3 días después de cada sesión de CAP.

En 11 casos (55\%) hubo mejoría satisfactoria del grado de oclusión y de los síntomas, de los cuales 5 presentaron mejoría después de la primera aplicación de CAP que se mantuvo durante el seguimiento, y 6 tuvieron mejoría sostenida después de 2-7 sesiones de CAP. De los pacientes que presentaron mejoría satisfactoria 5 recibieron dilatación traqueal en una o varias ocasiones. En 6 de 7 casos que presentaron mejoría satisfactoria fueron resecados granulomas. En 9 pacientes no hubo mejoría por no haber presentado reducción significativa del grado de oclusión; entre estos, 8 presentaron mejoría mínima de los síntomas o del grado de oclusión y en un caso de estenosis completa subglótica no hubo cambios. Las figuras 1 y 2 muestran ejemplos de casos con evolución satisfactoria y no satisfactoria respectivamente.

No hubo complicaciones relacionadas a la broncoscopia flexible o a la CAP. Hasta la conclusión del estudio, de los 9 casos con resultado no satisfactorio, 3 fueron sometidos a traqueoplastia, a uno se le colocó una prótesis de Montgomery en tráquea, 3 permanecen con traqueostomía y 2 cursan con vigilancia estrecha. El seguimiento promedio para todos los pacientes fue de $15.7 \pm 6.4$ meses (rango 7-30).
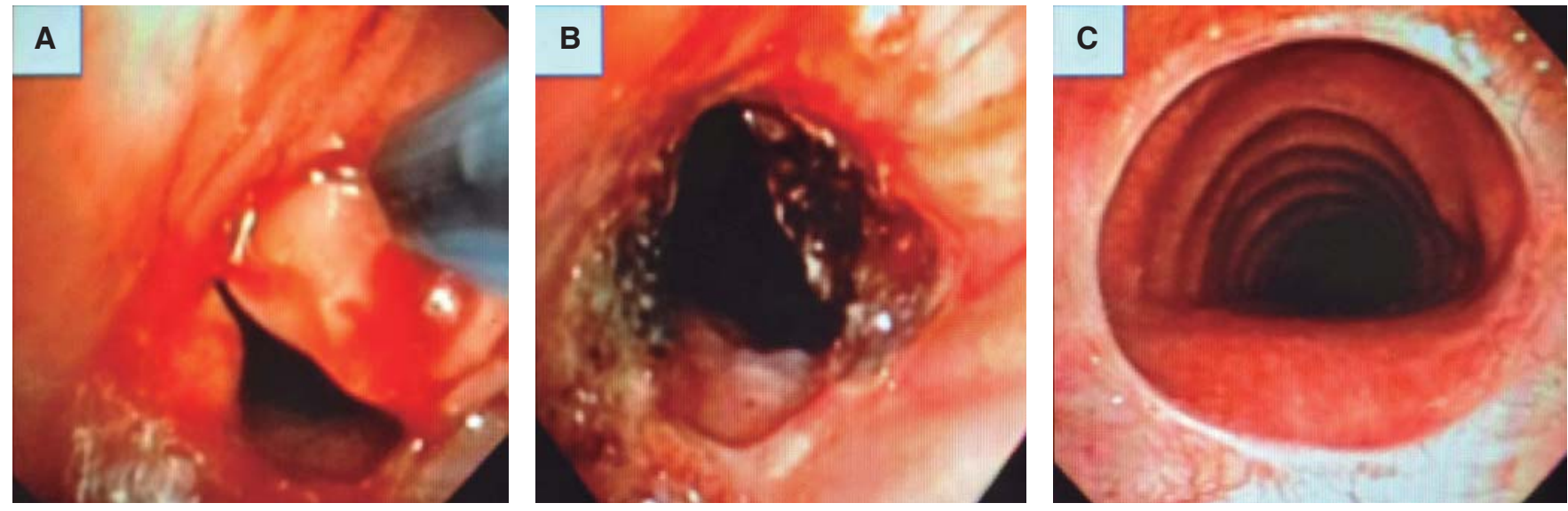

Figura 1. A. Paciente que presentó evolución satisfactoria. La obstrucción fue diagnosticada 44 días después de ser sometido a traqueoplastia con anastomosis traqueotraqueal. B. Inicialmente se resecaron tres granulomas, además se extrajo un punto de sutura. C. El paciente fue sometido a cuatro sesiones con uso de argón plasma y la recuperación del lumen se mantuvo estable después de 10.5 meses.
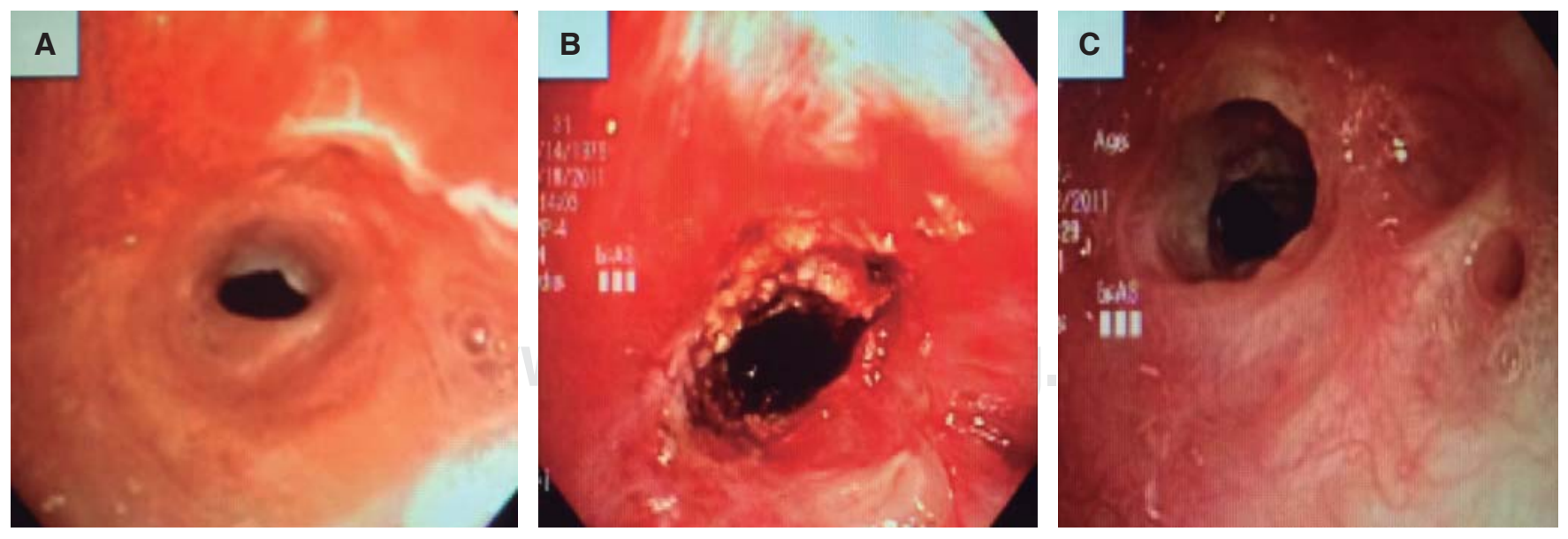

Figura 2. A. Caso de evolución no satisfactoria de un paciente con estenosis subglótica compleja después de intubación, a quien le fue colocado un stent de silicón endotraqueal retirado posteriormente por formación de granulomas. B. Fue sometido a una sesión de coagulación con argón plasma y dilatación mecánica. C. Último control endoscópico a los siete meses de seguimiento. 


\section{DISCUSIÓN}

Las lesiones endoluminales o de la pared de la vía aérea central que ocluyen su luz son diversas. El curso clínico de estas lesiones puede llevar a la obstrucción parcial con síntomas respiratorios limitantes, o a la obstrucción completa con riesgo de asfixia. Cuando la causa de obstrucción es benigna, se propone a la resección quirúrgica como el tratamiento de elección. ${ }^{4,8,11}$ El lugar que ocupan las modalidades de tratamiento endoscópico no está bien definido. Estas modalidades, solas o en combinación, han demostrado buenos resultados en casos seleccionados, ${ }^{5,16}$ de ahí que puedan ser consideradas como primera opción terapéutica. La decisión de tratamiento quirúrgico o endoscópico debe recaer siempre en un equipo experimentado de cirujanos y/o neumólogos intervencionistas en hospitales de referencia que atienden patología del tórax. ${ }^{5,8,9} \mathrm{La}$ elección entre los diferentes tipos de terapias endoscópicas ablativas dependerá de las características de la lesión, la disponibilidad de equipo y el entrenamiento y la experiencia del broncoscopista. ${ }^{12}$ En la actualidad existen varias modalidades de tratamiento endoscópico para la OBVA. ${ }^{14}$ Aunque la aplicación de CAP en OBVA fue inicialmente descrita en series de casos desde los $90,{ }^{17-19}$ todavía no existen ensayos clínicos que demuestren su utilidad o superioridad cuando se compara con otras técnicas.

Desde su introducción, el uso de láser Nd-YAG (del inglés neodynium: yttrium aluminium garnet) se convirtió en la técnica no quirúrgica más usada en el manejo de la obstrucción de la vía aérea por causa maligna, benigna y de alteraciones endobronquiales misceláneas, ${ }^{20-23}$ ante todo gracias a su poder para vaporizar tejidos y a su excelente capacidad de coagulación. ${ }^{12}$ La desventaja obvia del láser Nd-YAG sobre otros métodos para resección broncoscópica se hizo evidente cuando la relación costo-efectividad adquirió importancia. ${ }^{19,24} \mathrm{La}$ introducción de CAP en nuestra unidad de endoscopia torácica nos permitió prescindir del Nd-YAG logrando una tasa de éxito comparable a la obtenida con láser. 22,25 Con el uso de CAP, al igual que con láser y electrocauterio, hay un efecto de ablación del tejido inmediata, así como un efecto citocida retardado por transmisión de calor; sin embargo, su capacidad para vaporizar y carbonizar tejidos es menor.

Pudimos observar buenos resultados en lesiones vascularizadas como los granulomas, en que la terapia con CAP se recomienda. ${ }^{21,26,27} \mathrm{El}$ tratamiento con CAP en un caso de obstrucción subglótica del $100 \%$ no fue satisfactorio; las lesiones que involucran el cartílago cricoides y la membrana cricotiroidea incrementan el nivel de complejidad diagnóstico y terapéutico, ${ }^{28}$ y el tratamiento ablativo en estos casos puede ser susceptible de complicaciones fatales. ${ }^{29}$ En $60 \%$ de las sesiones de CAP se requirió de anestesia general cuando se realizó dilatación mecánica concomitante. Pensamos que el tratamiento adyuvante con dilatadores secuenciales tuvo un papel importante en los resultados ya que cinco de once pacientes que fueron sometidos a dilatación tuvieron un resultado satisfactorio.

Okiror et al..$^{30}$ combinaron dilatación y láser en el tratamiento de OBVA con buenos resultados, sin embargo, el seguimiento promedio fue de 7 meses. Si bien, la dilatación mecánica laringotraqueal con balón es la más empleada entre las técnicas de dilatación,,51-33 el uso del broncoscopio rígido, ${ }^{34}$ dilatadores esofágicos de Savary-Guilliard, ${ }^{35}$ dilatadores de Jackson, ${ }^{36}$ y el uso de cánulas de intubación orotraqueal de distintos tamaños también se han descrito; estas dos últimas técnicas son usadas de manera rutinaria en nuestra institución.

Hoy en día no existe certeza del beneficio que puedan ofrecer los corticosteroides ya sean inhalados, sistémicos o intralesionales y la mitomicina- $C$ para evitar la inflamación y re-oclusión postratamiento. ${ }^{14}$ En nuestra unidad de endoscopia torácica usamos esteroides sistémicos de manera rutinaria por su fácil administración y bajo costo.

Sobre todo se incluyó a pacientes con lesiones fibrosas con presencia de bridas y/o lesiones granulomatosas susceptibles de ser resecadas. La valoración de resultados tomada del expediente clínico y broncoscópico fue clínica y visual. El cambio en los síntomas postratamiento fue subjetivo ya que no se utilizó una escala; aunque, debió acompañarse de un cambio en el grado de oclusión de acuerdo a una escala previamente descrita..$^{15} \mathrm{En}$ los pacientes en que hubo mejoría satisfactoria, ésta se mantuvo durante su seguimiento; pero, considerando que el período de observación no fue a largo plazo (en promedio de 15 meses), la recidiva puede presentarse. Además de tratarse de un estudio retrospectivo, otra de las principales limitaciones de la serie de casos que se presenta es que incluyó a una población heterogénea de pacientes con OBVA por distintas etiologías; no obstante, consideramos que provee información útil acerca del uso de CAP en pacientes sintomáticos y su respuesta al tratamiento.

El número de series de casos que incluye entre otros métodos el uso de CAP para el manejo de la OBVA sigue creciendo, ${ }^{3,32}$ como también el de series de casos con uso de CAP como método primario, ${ }^{29,37,38} \mathrm{sin}$ embargo, es necesaria la elaboración de ensayos clínicos controlados que definan mejor el tipo de pacientes que se beneficiará más de esta técnica. 


\section{CONCLUSIONES}

La OBVA puede ser tratada de manera efectiva y segura con CAP a través de broncoscopia flexible en casos seleccionados. En esta serie según la definición usada, la CAP fue útil en $55 \%$ de los casos. La CAP puede considerarse una opción inicial de tratamiento, pudiendo obtenerse mejoría inmediata de la OBVA en diversos grados que en ocasiones puede ser definitiva. El manejo combinado con dilatadores puede ser de ayuda para disminuir el grado de obstrucción.

\section{REFERENCIAS}

1. Lorenz RR. Adult laryngotracheal stenosis: etiology and surgical management. Curr Opin Otolaryngol Head Neck Surg 2003;11(6):467-472.

2. García-Olazarán JG, Aguilar-Ramírez MP, Pérez-Romo AR, Navarro-Reynoso F, Cicero-Sabido R. Patología traqueal diagnosticada por fibrobroncoscopia. Experiencia en 111 casos. Rev Inst Nal Enf Resp Méx 2004;17(2):67-72.

3. Li YQ, Li Q, Bai C, et al. Causes of benign central airway stenoses and the efficacy of interventional treatments through flexible bronchoscopy. Zhonghua Jie He He Hu Xi Za Zhi 2008;31(5):364-368.

4. Marel M, Pekarek Z, Spasova I, et al. Management of benign stenoses of the large airways in the university hospital in Prague, Czech Republic, in 1998-2003. Respiration 2005;72(6):622-628.

5. Galluccio G, Lucantoni G, Battistoni $\mathrm{P}$, et al. Interventional endoscopy in the management of benign tracheal stenoses: definitive treatment at long-term follow-up. Eur J Cardiothorac Surg 2009;35(3):429-33. doi: 10.1016/j.ejcts.2008.10.041.

6. Yao XP, Li Q, Bai C, et al. Restenosis and its management after metallic stents implantation in benign tracheal and main bronchial stenosis. Zhonghua Nei Ke Za Zhi 2005;44(12):885-889.

7. Krajc T, Janík M, Lucenic M, Benej R, Harustiak S. The management of restenosis following segmental resection for postintubation tracheal injury. Rozhl Chir 2010;89(8):490-497.

8. Navarro-Reynoso FP, García-Sainz M, Peña-García JF, Cicero-Sabido R, Ibarra-Pérez C. Tratamiento multidisciplinario de la obstrucción de la vía aérea. Gac Med Mex 2002;138(5):427-443.

9. Peña J, Cicero R, Marín J, Ramírez M, Cruz S, Navarro F. Laryngotracheal reconstruction in subglottic stenosis: an ancient problem still present. Otolaryngol Head Neck Surg 2001;125(4):397-400.

10. Freitag L, Darwiche K. Endoscopic treatment of tracheal stenosis. Thorac Surg Clin 2014;24:27-40. doi: 10.1016/j. thorsurg.2013.10.003.

11. Rahman NA, Fruchter O, Shitrit D, Fox BD, Kramer MR. Flexible bronchoscopic management of benign tracheal stenosis: long term follow-up of 115 patients. J
Cardiothorac Surg 2010;5:2. doi: 10.1186/1749-80905-2.

12. Seaman JC, Musani Al. Endobronchial ablative therapies. Clin Chest Med 2013;34(3):417-25. doi: 10.1016/j. ccm.2013.04.006.

13. Park JH, Kim JH, Song HY, Shin JH, Ko HK. Management of benign tracheal strictures caused by tracheostomy. Cardiovasc Intervent Radiol 2014;37:743-9. doi: 10.1007/ s00270-013-0727-7.

14. Fernando HC, Sherwood JT, Krimsky W. Endoscopic therapies and stents for benign airway disorders: where are we, and where are we heading?. Ann Thorac Surg 2010;89(6):S2183S2187. doi: 10.1016/j.athoracsur.2010.02.106.

15. Freitag L, Ernst A, Unger M, Kovitz K, Marquette CH. A proposed classification system of central airway stenosis. Eur Respir J 2007;30(1):7-12.

16. Ciccone AM, De Giacomo T, Venuta F, et al. Operative and non-operative treatment of benign subglottic laryngotracheal stenosis. Eur J Cardiothorac Surg 2004;26(4):818-822.

17. Iwamoto $\mathrm{Y}$, Miyazawa $\mathrm{T}$, Kurimoto N, et al. Interventional bronchoscopy in the management of airway stenosis due to tracheobronchial tuberculosis. Chest 2004;126(4):1344-1352.

18. Yasuo M, Tanabe T, Tsushima K, et al. Endobronchial argon plasma coagulation for the management of post-intubation tracheal stenosis. Respirology 2006;11(5):659-662.

19. Avanesian AA, Shcherbakov AM, Arsen'ev Al. An experience with argon-plasma coagulation in endoscopic management of obstructive tumors of the trachea and main bronchi. Vopr Onkol 2004;50(4):459-461.

20. Navarro-Reynoso FP, Pérez-Romo AR, Cicero-Sabido R. Resección endoscópica con laser Nd-YAG de lesiones benignas y malignas traqueales (reporte de 2 casos). Gac Med Mex 1994;130:S44.

21. Bolliger CT, Mathur PN, Beamis JF, et al.; European Respiratory Society/American Thoracic Society. ERS/ATS statement on interventional pulmonology. European Respiratory Society/American Thoracic Society. Eur Respir J 2002;19(2):356-373.

22. Navarro-Reynoso FP, Pérez-Romo A, Green-Schneeweiss L. Consideraciones sobre el uso del láser Nd:YAG en neumología. Cir Ciruj 2004;72(5):369-373.

23. Vázquez CJ, Navarro RF, Pérez RA, Rentería PN, Cicero SR. Resección de lesiones traqueobronquiales con broncoscopia Nd:YAG. Estudio preliminar. Neumol Cir Torax 1997;53(4):92-98.

24. Boxem Tv, Muller M, Venmans B, Postmus P, Sutedja T. Nd-YAG laser vs. bronchoscopic electrocautery for palliation of symptomatic airway obstruction: a costeffectiveness study. Chest 1999;116(4):1108-1112.

25. Navarro RF, Vazquez CJ, Cicero SR. Laser resection of benign obstructive lesions of the airway. Chest 1998;114(Suppl):304s.

26. Sato M, Terada Y, Nakagawa T, Li M, Wada H. Successful use of argon plasma coagulation and tranilast to treat granulation tissue obstructing the airway after tracheal anastomosis. Chest 2000;118(6):1829-1831. 
27. Keller CA, Hinerman R, Singh A, Alvarez F. The use of endoscopic argon plasma coagulation in airway complications after solid organ transplantation. Chest 2001;119(6):1968-1975.

28. Stoelben E, Koryllos A, Beckers F, Ludwig C. Benign stenosis of the trachea. Thorac Surg Clin 2014;24(1):59-65. doi: 10.1016/j.thorsurg.2013.09.001.

29. Jin FG, Mu DG, Chu DL, et al. Efficacy and safety of argon plasma coagulation in the treatment of obstructive tracheobronchial stenosis. Zhonghua Zhong Liu Za Zhi 2008;30(6):462-464.

30. Okiror L, Jiang L, Oswald N, et al. Bronchoscopic management of patients with symptomatic airway stenosis and prognostic factors for survival. Ann Thorac Surg 2015;99(5):1725-1730. doi: 10.1016/j.athoracsur.2015.01.061.

31. Lee KH, Ko GY, Song HY, Shim TS, Kim WS. Benign tracheobronchial stenoses: long-term clinical experience with balloon dilation. J Vasc Interv Radiol 2002;13(9 Pt 1):909-914.

32. Jin FG, Fu EQ, Xie YH, et al. The application of combined interventional procedures for the management of intractable central airway stenosis. Zhonghua Jie $\mathrm{He} \mathrm{He}$ Hu Xi Za Zhi 2010;33(1):21-24.

33. Liang $\mathrm{YL}$, Liu GN, Zheng HW, et al. Management of benign tracheal stenosis by small-diameter tubeassisted bronchoscopic balloon dilatation. Chin Med J 2015;128(10):1326-1330. doi: 10.4103/03666999.156776
34. Madan K, Agarwal R, Aggarwal AN, Gupta D. Therapeutic rigid bronchoscopy at a tertiary care center in North India: Initial experience and systematic review of Indian literature. Lung India 2014;31(1):9-15. doi: 10.4103/0970-2113.125887.

35. Chang AC, Pickens A, Orringer MB. Awake tracheobronchial dilation without the use of rigid bronchoscopy. Ann Thorac Surg 2006;82(6):e43-45.

36. Alvarez-Maldonado P, Peña J, Criales-Cortés JL, NavarroReynoso F, Pérez-Romo A, Cicero-Sabido R. Benign tracheal stenosis: a case series analysis. J Bronchology Interv Pulmonol 2009;16(4):254-260. doi: 10.1097/ LBR.0b013e3181b61579.

37. Dang BW, Zhang J. Safety and efficacy of argon plasma coagulation for resection of lipomas and hamartomas in large airways. Asian Pac J Cancer Prev 2011;12(2):477-480.

38. Miller SM, Bellinger CR, Chatterjee A. Argon plasma coagulation and electrosurgery for benign endobronchial tumors. J Bronchology Interv Pulmonol 2013;20(1):3840. doi: 10.1097/LBR.0b013e318282d3ca.

\section{$\triangle$ Correspondencia:}

Dr. Carlos Núñez-Pérez Redondo, Servicio de Neumología y Cirugía de Tórax, Hospital General de México "Dr. Eduardo Liceaga". Dr. Balmis 148, colonia Doctores, Del. Cuauhtémoc, 06726, Ciudad de México.

Correo electrónico: drnunezpr@icloud.com

Los autores declaran no tener conflicto de intereses. 